\title{
Construction and Performance Analysis of BIBD-QC-LDPC Codes for Correcting Erasure-Bursts
}

\author{
Sheng Huang, Xueting Jia, Jianguo Yuan and Pan $\mathrm{Mu}$ \\ Key Laboratory of Optical Fiber Communication Technology, Chongqing \\ University of Posts and Telecommunications, Chongqing, 400065, China \\ huangs@cqupt.edu.cn; cquptjxt@163.com; yuanjg@cqupt.edu.cn; \\ cquptmp@163.com
}

\begin{abstract}
This paper presents a novel approach for constructing structured regular QC-LDPC codes based on a special type of combinatorial designs, known as the balanced incomplete block design (BIBD).The code design approach based on the technology of block cyclic shift and dispertion (BCSD) for correcting erasure-bursts over the binary bursts erasure channel (BBEC). Furthermore, the Tanner graph of the constructed codes is free of 4cycles, hence the BCSD-QC-LDPC codes have girth at least 6 . The simulation results show that the BCSD-QC-LDPC codes perform well over the BBEC channel with iterative message-passing decoding.
\end{abstract}

Keywords: quasi-cyclic low-density parity-check $(Q C$ - LDPC) code, balanced incomplete block design (BIBD), blocks cyclic shift and dispersion (BCSD), binary bursts erasure channel (BBEC)

\section{Introduction}

Recently, in order to improve the quality and reliability of the communication system, forward error correction (FEC) technology [1] is widely used in various fields of information processing. There were various kinds of error correcting codes, such as Hamming codes, BCH codes, RS codes and low-density parity-check (LDPC) codes [2]. Quasi-cyclic low-density parity-check (QC-LDPC) codes are of particular interest [3-5] by virtue of its lower encoding complexity and simpler hardware implementation capacity compared with the random LDPC codes [6,7]. Generally speaking, the correcting position of error correcting code is unknown in advance. But for a special erasure technique, receivers are able to detect the presence of these time intervals and may choose, accordingly, to erasure some or all of the symbols received during such intervals. This erasure technique causes symbol losses at known locations. Therefore the erasure code can be processed easier than the error correcting code. The erasure codes which adopt the erasure channel model [8] are also known as loss resilient codes [9]. Over a binary erasure channel (commonly called BEC) [10], erasures occur at random. However, there is another type of binary erasure channel over which erasures cluster into bursts. A channel of this type is called a binary burst erasure channel (BBEC)[11]. In order to resist such burst erasure effectively in high-speed data transmission system, it is very important to find QC-LDPC codes for correcting erasure-bursts.

Combinatorial design theory is a rich field of powerful tools for constructing LDPC codes. And balanced incomplete block design (BIBD) [12] as a branch of combinatorial design has been widely used for constructing LDPC codes. For instance, there has been three types of methods for constructing LDPC codes, such as the traditional method [13], the improved method based on the decomposition technique [14-15] which can reduce the density of the parity check matrix and hence reduces the number of the short cycles, and the array dispersion (AD) method which can construct good LDPC codes for correcting 
erasure bursts [11]. In this paper, we present a novel method for constructing QC-LDPC codes based on block cyclic shift and dispersion (BCSD).The main idea of BCSD is let the CPMs of BIBD-QC-LDPC codes be portioned into blocks and then disperses them in some way. The codes constructed by the $\mathrm{AD}$ and the codes constructed by the BCSD are referred to as AD-QC-LDPC codes and BCSD-QC-LDPC respectively. The shortest code length of BCSD-QC-LDPC codes are little shorter than the AD-QC-LDPC codes with same code rate. Moreover, the zero-covering-span of BCSD-QC-LDPC codes is bigger than the AD-QC-LDPC codes with same code rate. The parity check matrices of BCSDQC-LDPC codes satisfy the RC-constraints, therefore the associated Tanner graph is free of cycles of length 4 and the resultant code has the minimum distance equal to the column weight plus one. The simulation results show that the BCSD-QC-LDPC codes perform well over the BBEC channel with iterative message-passing decoding.

\section{Review of BIBD-LDPC Codes}

\subsection{Balanced Incomplete Block Design}

In this section, we give some basic concepts of balanced incomplete block design.

A ( $m, n, r, c, \lambda)$-BIBD satisfies the following conditions: 1) Let $\boldsymbol{X}=\left\{x_{1}, x_{2}, \ldots, x_{m}\right\}$ be a set of $m$ elements; 2)Let $\boldsymbol{B}=\left\{B_{1}, B_{2}, \ldots, B_{n}\right\}$ be a collection of nonempty subsets of $\boldsymbol{X}$;3)each object $x_{i}(1 \leq i \leq m)$ appears in exactly $r$ of the $n$ blocks;4)each block contains exactly $c$ object;5)every two objects $x_{i}, x_{j}(1 \leq i \leq m, 1 \leq j \leq m, \quad i \neq j)$ appear together in exactly $\lambda$ of the $n$ blocks[12].

Meanwhile, a $(m, n, r, c, \lambda)$-BIBD can be completely represented by its incidence matrix [12] $\boldsymbol{H}=\left[h_{i j}\right]$, based on the following definitions:

$$
h_{i j}= \begin{cases}0 & \text { if } x_{i} \notin \mathrm{B}_{j} \\ 1 & \text { if } x_{i} \in \mathrm{B}_{j}\end{cases}
$$

The incidence matrix of a $(m, n, r, c, \lambda)$-BIBD satisfies the following properties: 1) row weight of $\boldsymbol{H}$ is $r ; 2$ ) column weight of $\boldsymbol{H}$ is $c ; 3$ )two distinct rows of $\boldsymbol{H}$ both contain " 1 "s in exactly $\lambda$ of n columns [12].

Theorem 1: A $(m, c, \lambda)$-BIBD, every object $x_{i}(1 \leq i \leq m)$ appears in $r=\lambda(m-1) /(c-1)$ of $\mathrm{n}$ blocks.

Theorem 2: A $(m, c, \lambda)$-BIBD have exactly $n=m r / c=\lambda\left(m^{2}-m\right) /\left(c^{2}-c\right)$ blocks.

Eaxmple1: A $(9,3,1)$-BIBD , $X=\{1,2,3,4,5,6,7,8,9\}$, $B=\{123,456,789,147,258,369,159,267,348,168,249,357\}$ for $m=9, c=3, \lambda=1$, according to theorem 1 and theorem 2 , we can obtained,

$$
\begin{gathered}
r=\lambda(m-1) /(c-1)=1 \times(9-1) /(3-1)=4 \\
n=m r / c=\lambda\left(m^{2}-m\right) /\left(c^{2}-c\right)=1 \times\left(9^{2}-9\right) /\left(3^{2}-3\right)=12
\end{gathered}
$$

its incidence matrix is expressed as (2).

$$
H=\left[\begin{array}{llllllllllll}
1 & 0 & 0 & 1 & 0 & 0 & 1 & 0 & 0 & 1 & 0 & 0 \\
1 & 0 & 0 & 0 & 1 & 0 & 0 & 1 & 0 & 0 & 1 & 0 \\
1 & 0 & 0 & 0 & 0 & 1 & 0 & 0 & 1 & 0 & 0 & 1 \\
0 & 1 & 0 & 1 & 0 & 0 & 0 & 0 & 1 & 0 & 1 & 0 \\
0 & 1 & 0 & 0 & 1 & 0 & 1 & 0 & 0 & 0 & 0 & 1 \\
0 & 1 & 0 & 0 & 0 & 1 & 0 & 1 & 0 & 1 & 0 & 0 \\
0 & 0 & 1 & 1 & 0 & 0 & 0 & 1 & 0 & 0 & 0 & 1 \\
0 & 0 & 1 & 0 & 1 & 0 & 0 & 0 & 1 & 1 & 0 & 0 \\
0 & 0 & 1 & 0 & 0 & 1 & 1 & 0 & 0 & 0 & 1 & 0
\end{array}\right]_{9 \times 12}
$$




\subsection{BIBD-LDPC Codes}

There are three types of BIBD-LDPC codes based on BIBD, namely BIBD-LDPC codes constructed from prime fields GF $(6 t+1)$ presented by Netto and BIBD-LDPC codes constructed from prime fields $\mathrm{GF}(12 t+1)$ and $\mathrm{GF}(20 t+1)$ presented by Bose. They can be represented as $\mathrm{GF}(m), m$ for $6 t+1,12 t+1$ and $20 t+1$. Let $t$ be a positive integer such that $m$ is a prime. Then there exists a prime field $\operatorname{GF}(m)=\{0,1,2, \ldots, m$ 1 , let $\alpha$ be a primitive element of $\operatorname{GF}(m)$ such that the powers of $\alpha, \alpha^{-\infty}=0, \alpha^{0}=1$, $\alpha^{1}, \ldots, \alpha^{m-2}$, give all the $m$ elements of $\operatorname{GF}(m)$ and $\alpha^{m-1}=1$.

For instance, to form a Netto BIBD-LDPC codes, we first form $t$ base blocks: $B_{i, 0}=\left\{\alpha^{i}, \alpha^{i+2 t}, \alpha^{i+4 t}\right\},(0 \leq i \leq t-1)$. For each base block $B_{i, 0}$, we form $6 t+1$ blocks, $B_{i, 0}$, $B_{i, 1}, \ldots, B_{i, 6 t}$, by adding each element of $\mathrm{GF}(6 t+1)$ in turn to the elements in $B_{i, 0}$. Then,for $0 \leq j \leq 6 t, B_{i, j}=\left\{j+\alpha^{i}, j+\alpha^{i+2 t}, j+\alpha^{i+4 t}\right\}$, can be represented by its incidence matrix $\boldsymbol{Q}_{i}$ as $\boldsymbol{H}_{0}=\left[\begin{array}{llllll}\boldsymbol{Q}_{0} & \boldsymbol{Q}_{1} & \boldsymbol{Q}_{2} & \ldots & \boldsymbol{Q}_{t-1}\end{array}\right] \cdot \boldsymbol{H}_{0}$ is the parity check matrix of BIBD-QCLDPC codes, and the above construction method of QC-LDPC codes based on BIBD is called traditional method. $\boldsymbol{H}_{0}$ is a $(6 t+1) \times t(6 t+1)$ matrix with row weight $3 t$, column weight 3 , and density $3 /(6 t+1)$. It is very small when $t \geq 4$, and since $\lambda=1$, no two rows or two columns of $\boldsymbol{H}_{0}$ have more than one position that has the same 1 (RC-constraint)[15]. Apparently, $\boldsymbol{H}_{0}$ is composed of $t$ CPMs of $(6 t+1) \times(6 t+1)$. Consequently, the parity check matrices constructed from the method all satisfy the conditions of QC-LDPC codes' parity check matrices. The null space of $\boldsymbol{H}_{0}$ gives a BIBD-QC-LDPC code of length $t(6 t+1)$, and the Tanner graph has no girth 4 .

As for $\boldsymbol{H}_{0}$, where row or column weights of each $\boldsymbol{Q}_{i}$ is 3 .Each circulant, $\boldsymbol{Q}_{i}(0 \leq i \leq t-$ 1),can be decomposed[16] into 3 component circulants, denoted as $\boldsymbol{A}_{0, i}, \boldsymbol{A}_{1, i}, \boldsymbol{A}_{2, i}$. the following $3 \times t$ array of $(6 t+1) \times(6 t+1)$ CPMs is obtained:

$$
\boldsymbol{H}_{1}=\left[\begin{array}{cccc}
\boldsymbol{A}_{0,0} & \boldsymbol{A}_{0,1} & \cdots & \boldsymbol{A}_{0, t-1} \\
\boldsymbol{A}_{1,0} & \boldsymbol{A}_{1,1} & \cdots & \boldsymbol{A}_{1, t-1} \\
\boldsymbol{A}_{2,0} & \boldsymbol{A}_{2,1} & \cdots & \boldsymbol{A}_{2, t-1}
\end{array}\right]
$$

$\boldsymbol{H}_{1}$ is a $3(6 t+1) \times t(6 t+1)$ matrix with row weight $t$, column weight 3 and density 1/ $(6 t+1)$. The null space of $\boldsymbol{H}_{1}$ gives a BIBD-QC-LDPC codes of length $t(6 t+1)$, and the Tanner graph has no girth $4 . \boldsymbol{H}_{1}$ is also called the completely decomposed parity check matrix based on decomposition technique.

Three types of BIBD-QC-LDPC codes can be designed, as shown in Table 1.

Table 1. Three Types of BIBD-QC-LDPC Codes

\begin{tabular}{|c|c|c|c|c|c|c|}
\hline Ty & $\begin{array}{l}\text { Galois } \\
\text { Field }\end{array}$ & Baseblock & Primitive element $\alpha$ & $m$ & $n$ & \\
\hline $\mathrm{N}$ & $\mathrm{GF}(6 \mathrm{t}+$ & $B_{i}=\left\{\alpha^{i}, \alpha^{i-2 t}, \alpha^{i-4 t}\right\}$ & $a \in \mathrm{GF}(6 \mathrm{t}-1)$ & $6 t$ & $t(6 t+$ & \\
\hline etto & 1) $\mathrm{GF}(12 \mathrm{t}$ & $B=\left\{0, \alpha^{2 i}, a^{2 i-4 t}, \alpha^{2 i} t^{-8 t}\right\}$ & $a^{4 t}-1=\alpha^{x} x \leq 12 t+1, x$ is a positive & +1 & 1) $t(12 t$ & $t$ \\
\hline ose- & $+1)$ & & ood integer & $t+1$ & $+1)$ & $t$ \\
\hline $\begin{array}{c}\text { B } \\
\text { ose- } \\
\text { II }\end{array}$ & ${ }_{+1)} \mathrm{GF}(20 t$ & $\begin{aligned} & B_{i}=\left\{\alpha^{2 i}, \alpha^{2 i-4 t}, a^{2 i+8 t},\right. \\
&\left.\alpha^{2 i+12 t} ; a^{2 i-16 t}\right\}\end{aligned}$ & $\begin{array}{l}\alpha^{4}+1=\alpha^{x}, x \leq 20 t-1, x \text { is a positive } \\
\text { ood integer }\end{array}$ & $t+1$ & $+1)^{t(20 t}$ & $t$ \\
\hline
\end{tabular}




\section{Novel QC-LDPC Codes for Correcting Erasure Bursts}

The RC-constrained arrays of circulant permutation matrices constructed based on GF $(12 t+1)$ is expressed as (4):

$$
\boldsymbol{H}=\left[\begin{array}{llll}
\boldsymbol{A}_{0,0} & \boldsymbol{A}_{0,1} & \cdots & \boldsymbol{A}_{0, t-1} \\
\boldsymbol{A}_{1,0} & \boldsymbol{A}_{1,1} & \cdots & \boldsymbol{A}_{1, t-1} \\
\boldsymbol{A}_{2,0} & \boldsymbol{A}_{2,1} & \cdots & \boldsymbol{A}_{2, t-1} \\
\boldsymbol{A}_{3,0} & \boldsymbol{A}_{3,1} & \cdots & \boldsymbol{A}_{3, t-1}
\end{array}\right]
$$

To illustrate the code construction by BCSD, first, choose the CPMs of the first row to the $z(2 \leq z \leq 4)$ row of $\boldsymbol{H}$. Then partition it into $j$ disjoint blocks by columns, where $j=\lfloor(t+1) / z\rfloor$, as shown in (5). For $1 \leq i \leq j, j \in Z^{+}, \boldsymbol{H}_{i}(z, z)$ is consist of $z$ columns which are between the $(i-1) z+1$ column and $i z$ column of $\boldsymbol{H}$, as shown in (6).

$$
\boldsymbol{H}=\left[\begin{array}{cccc:ccccccc} 
& \text { block } 1 & & & \multicolumn{1}{c}{\text { block } 2} & & & \cdots & \cdots \\
A_{0,0} & A_{0,1} & \cdots & A_{0, z-1} & A_{0, z} & A_{0, z+1} & \cdots & A_{0,2}: 1 & \cdots & A_{0, t-1} \\
A_{1,0} & A_{1,1} & \cdots & A_{1, z-1} & A_{1, z} & A_{1, z+1} & \cdots & A_{1,2} & \cdots & A_{1, t-1} \\
\vdots & \vdots & \ddots & \vdots & \vdots & \vdots & \ddots & \vdots & \ddots & \vdots \\
\hdashline A_{z-1,0} & A_{z-1,1} & \cdots & A_{z-1, z-1} & A_{z-1, z} & A_{z-1, z+1} & \cdots & A_{z-1,2 z-1} & \cdots & A_{z-1, t-1} \\
\vdots & \vdots & \ddots & \vdots & \vdots & \vdots & \ddots & \vdots & \ddots & \vdots \\
A_{3,0} & A_{3,1} & \cdots & A_{3, z-1} & A_{3, z} & A_{3, z+1} & \cdots & A_{3,2 z-1} & \cdots & A_{3, t-1}
\end{array}\right]
$$

$$
\boldsymbol{H}_{i}(z, z)=\left[\begin{array}{cccc}
\boldsymbol{A}_{0, z(i-1)} & \boldsymbol{A}_{0,1+z(i-1)} & \cdots & \boldsymbol{A}_{0, z-1+z(i-1)} \\
\boldsymbol{A}_{1, z(i-1)} & \boldsymbol{A}_{1,1+z(i-1)} & \cdots & \boldsymbol{A}_{1, z-1+z(i-1)} \\
\vdots & \vdots & \ddots & \vdots \\
\boldsymbol{A}_{z-1, z(i-1)} & \boldsymbol{A}_{z-1,1+z(i-1)} & \cdots & \boldsymbol{A}_{z-1, z-1+z(i-1)}
\end{array}\right]
$$

Secondly, we obtain the sub matrices $\boldsymbol{H}_{1 B C S D}(z q, z q) \ldots \boldsymbol{H}_{i B C S D}(z q, z q) \ldots \boldsymbol{H}_{j B C S D}(z q$, $z q)$ from $\boldsymbol{H}_{1}(z, z) \ldots \boldsymbol{H}_{i}(z, z) \ldots \boldsymbol{H}_{j}(z, z)$ by the BCSD, where $q$ is the number of cyclicshift for every block, $q \geq 1$ and $q \in \mathrm{Z}^{+}$. The main idea of BCSD method is described by the following instance.

For instance, take $z=4, \boldsymbol{H}_{i \text { BCSD }}(4 q, 4 q)$ is shown in (7). Every 4 rows portioned by dash lines in $\boldsymbol{H}_{i \mathrm{BCSD}}(4 q, 4 q)$ is called as a block. The non-zero matrices of first block is corresponding to the first row of $\boldsymbol{H}_{i}(4,4)$, the second row of first block is a right parallelshift of the second row of $\boldsymbol{H}_{i}(4,4)$, the third row of first block is two right parallel-shift of the third row of $\boldsymbol{H}_{i}(4,4)$, and the fourth row of first block is three right parallel-shift of $\boldsymbol{H}_{i}(4,4)$. After filling the non-zero matrices of the 4 rows, filling the rest of the blank spaces with all zero matrices $\boldsymbol{O}$. The rest of $q$-1 blocks in $\boldsymbol{H}_{i \text { BCSD }}(4 q, 4 q)$ is expressed as (7). 


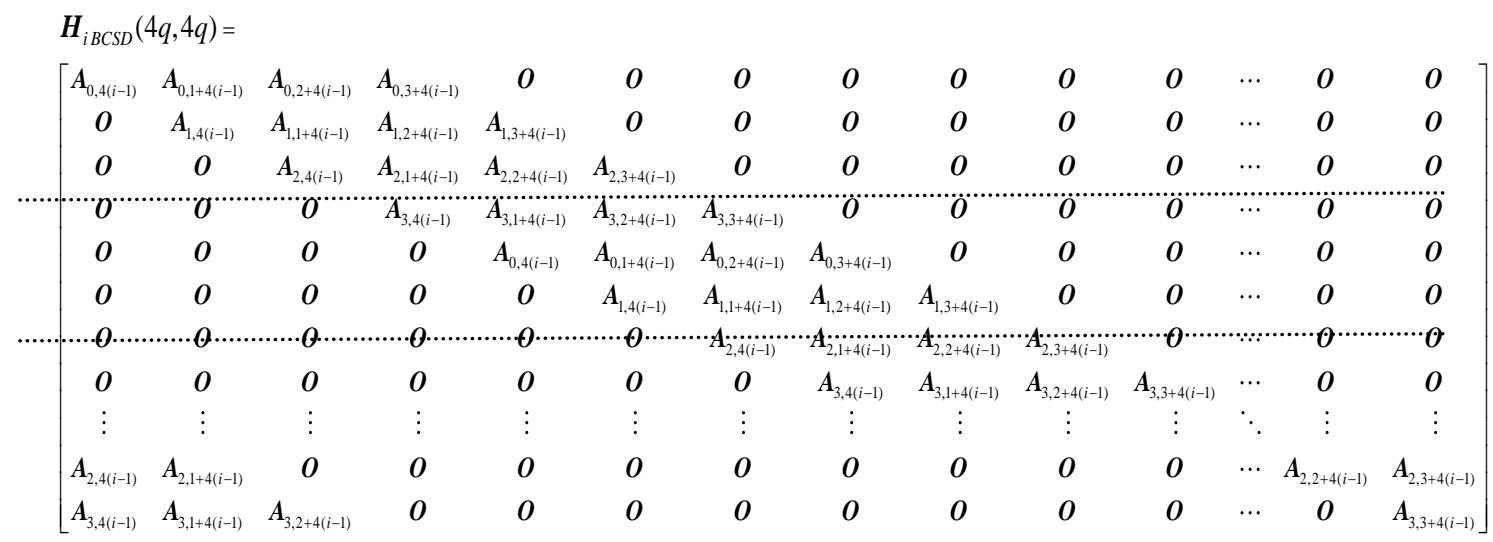

Finally, we take $k$ sub matrices $\boldsymbol{H}_{1 \mathrm{BCSD}}(4 q, 4 q) 、 \boldsymbol{H}_{2 \mathrm{BCSD}}(4 q, 4 q) \ldots \boldsymbol{H}_{k \mathrm{BCSD}}(4 q, 4 q)$, $1 \leq k \leq j$, we can obtain $\boldsymbol{H}_{k}$ :

$$
\boldsymbol{H}_{k}=\left[\boldsymbol{H}_{1 \mathrm{BCSD}}(4 q, 4 q) \boldsymbol{H}_{2 \mathrm{BCSD}}(4 q, 4 q) \boldsymbol{H}_{k \mathrm{BCSD}}(4 q, 4 q)\right]
$$

Because $\boldsymbol{H}$ satisfies the RC constraints, Tanner graph has no girth $4, \boldsymbol{H}_{k}$ also has no girth 4, with row weight $k z$, column weight $z$. The length of the zero-covering-span[14] of $\boldsymbol{H}_{i, \mathrm{BCSD}}(q z, q z)$ is at least $(q-1) z \times(12 t+1)$, indicating that it can correct the erasurebursts at least $(q-1) z \times(12 t+1)+1$.Compare the BCSD with the $\mathrm{AD}$ about the shortest code length with same code rate based on $\mathrm{GF}(12 t+1)$. Take $z=4$, the results is shown in Table 2. From Table 2, we can see that the shortest code length from the BCSD is far less than the shortest code length from the $\mathrm{AD}$ with same code rate obviously. This shows that the structures of the constructed BCSD-QC-LDPC codes are more flexible in selecting the code length.

Table 2. Comparison of the Shortest Code Length at the Same Code Rate

\begin{tabular}{ccc}
\hline Code rate & AD method & BCSD method \\
\hline $1 / 2$ & $\geqq 3664$ & $\geqq 1552$ \\
$2 / 3$ & $\geqq 8088$ & $\geqq 4344$ \\
$3 / 4$ & $\geqq 13088$ & $\geqq 7328$ \\
$4 / 5$ & $\geqq 21640$ & $\geqq 9640$ \\
$5 / 6$ & $\geqq 34032$ & $\geqq 16176$ \\
\hline
\end{tabular}

Table3. Comparison of Length of the Zero-Covering-Span of the Codes with Similar Code Length

\begin{tabular}{cccccc}
\hline Code rate & \multicolumn{1}{c}{1} & $2 /$ & $3 / 4$ & $4 / 5$ & $5 / 6$ \\
\hline Code length of AD method & 3 & 8 & 13 & 216 & 34 \\
& 664 & 088 & 088 & 40 & 032 \\
zero-covering-span of the AD-QC-LDPC & 9 & 1 & 16 & 216 & 28 \\
codes & 16 & 348 & 36 & 4 & 36 \\
Code length of BCSD method & 3 & 6 & 10 & 166 & 29 \\
& 488 & 516 & 992 & 20 & 448 \\
zero-covering-span of the BCSD-QC-LDPC & 1 & 1 & 18 & 221 & 32 \\
codes & 308 & 448 & 32 & 6 & 72 \\
\hline
\end{tabular}

It is seen from Table 3 that the zero-covering-span of the BCSD-QC-LDPC codes is bigger than the AD-QC-LDPC codes with same code rate. That is to say, the BCSD-QCLDPC codes have stronger ability for correcting erasure-bursts. 


\section{Simulation Results and Analysis}

Example2. Let $t=18, \alpha=3$ define a Netto $(109,1962,54,3,1)$-BIBD. If we take $z=3, q=2, k=6$, form a BCSD-QC-LDPC $(3924,3270)$ code by BCSD with code rate 0.833 . The zero-covering-span of $\boldsymbol{H}$ is at least $(q-1) z \times(6 t+1)=327$, indicating that it can correct the erasure-bursts at least 328. The error correcting performance of this code over the BBEC is shown in Fig.1. The BCSD-QC-LDPC $(3924,3270)$ code performs 0.045 from the Shannon limit at the Unresolved-error-bit-rate (UEBR) of $10^{-7}$. We also see that BCSD-QC-LDPC $(3924,3270)$ code performs better than the BIBD-QC-LDPC $(4254,3545)$ code by traditional method and the BIBDdes-QC-LDPC $(4206,3505)$ code based on decomposition technique (zerocovering-span of the two codes are also less than 327 ).

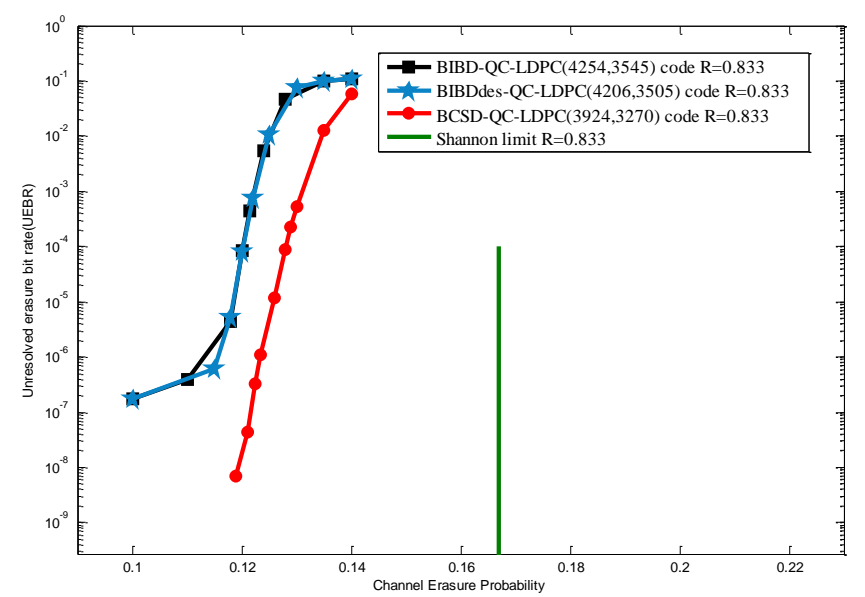

Figure 1. Performance of BCSD-QC-LDPC $(3924,3270)$ Code for BBEC, Code Rate $=0.833$

Let $t=21, \alpha=3$ define a Netto $(127,2667,63,3,1)$-BIBD. We construct a BCSDQC-LDPC $(4572,3810)$ code with code rate 0.833 by taking $z=3, q=2, k=6$. The zero-covering-span of $\boldsymbol{H}$ is at least $(q-1) z \times(6 t+1)=381$, indicating that it can correct the erasure-bursts at least 382. Let $t=18, \alpha=3$, if we take $z=3, q=3, k=6$, we construct a BCSD-QC-LDPC $(5886,4905)$ code with code rate 0.833 . The zero-covering-span of $\boldsymbol{H}$ is at least $(q-1) z \times(6 t+1)=654$, indicating that it can correct the erasure-bursts at least 655 .

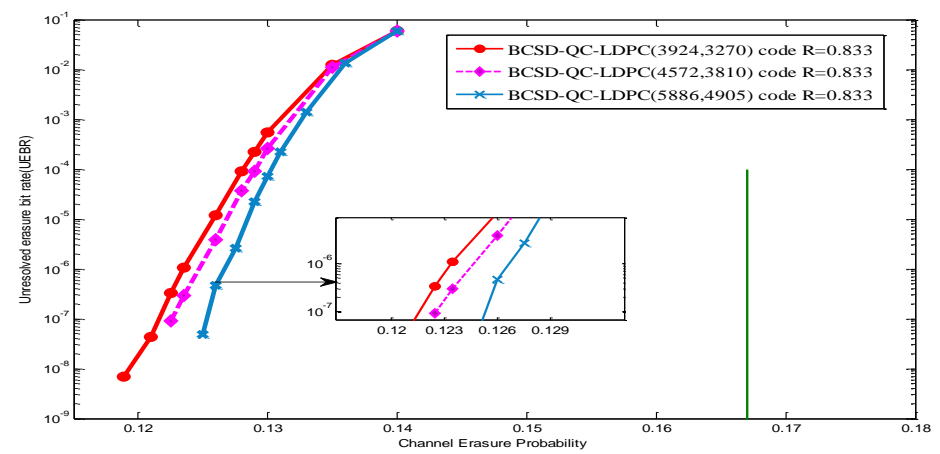

Figure 2. Performance of BCSD-QC-LDPC Code for BBEC, Code
Rate $=0.833$

From Figure 2, the error correcting performances of three codes are also superior, and they converge very fast. 
Example3. Let $t=18$ and $\alpha=3$, then $m=6 t+1=109, n=t \quad(6 t+1)=1962$ and $r=18 \times 3=54, c=3$. Based on $(109,1962,54,3,1)-\mathrm{BIBD}$, we use the BCSD method to construct a regular BCSD-QC-LDPC $(7848,6540)$ code with code rate 0.833 by taking $z=3, q=4$ and $k=6$. The zero-covering-span of $\boldsymbol{H}$ is at least $(q-1) z \times(6 t+1)=981$ and which is capable of correcting any erasure-burst of length up to 982.Let $t=27$ and $\alpha=2$, then $m=6 t+1=163, \quad n=t(6 t+1)=4401$ and $\quad r=27 \times 3=81, \quad c=3$. Based on $(163,4401,81,3,1)$-BIBD, take $z=3, q=3, k=6$, we use the BCSD method to construct a regular BCSD-QC-LDPC $(8802,7335)$ code with code rate 0.833 which is capable of correcting any erasure-burst of length up to 979 . We compare above two codes with the AD-QC-LDPC $(8028,6690)$ code constructed by the AD method at the same code rate 0.833 ( zero-covering-span of the AD-QC-LDPC $(8028,6690)$ code is at least 669). The error performance of the three codes over the BBEC is shown in Fig.3. The BCSD-QC-LDPC $(7848,6540)$ code and BCSD-QC-LDPC $(8802,7335)$ code perform better than AD-QC-LDPC $(8028,6690)$ code.The coding and decoding complexity is in direct proportion to code length $(O(n))$, therefore the coding and decoding complexity of BCSD-QC-LDPC $(7848,6540)$ code is decreased to some extent compared with AD-QC-LDPC $(8028,6690)$ code.

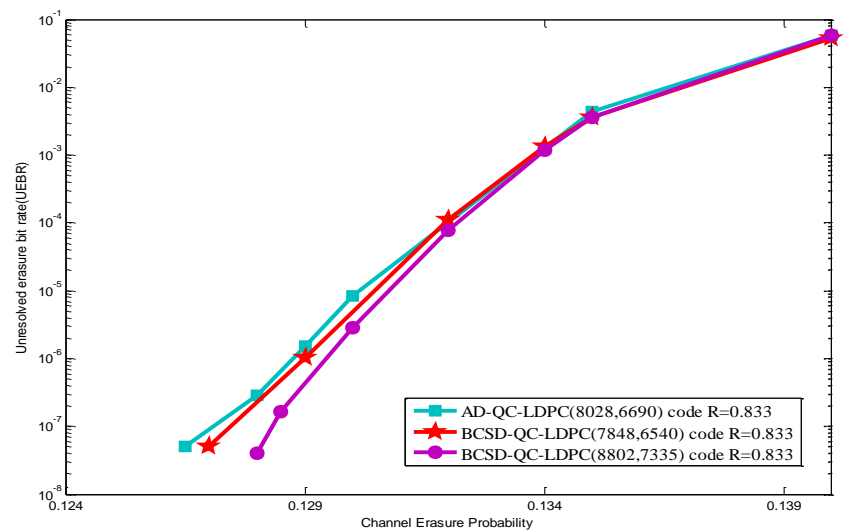

Figure 3. Performance of BCSD-QC-LDPC Code Compared with AD-QCLDPC Code for BBEC, Code Rate $=\mathbf{0 . 8 3 3}$

In the same way, we construct BCSD-QC-LDPC $(3488,1744)$ code with zerocovering-span 1308 and AD-QC-LDPC $(3664,1832)$ code with zero-covering-span 916 with same code rate 0.5. From Figure 4, we can see that BCSD-QC-LDPC code performs better than AD-QC-LDPC $(3664,1832)$ code.

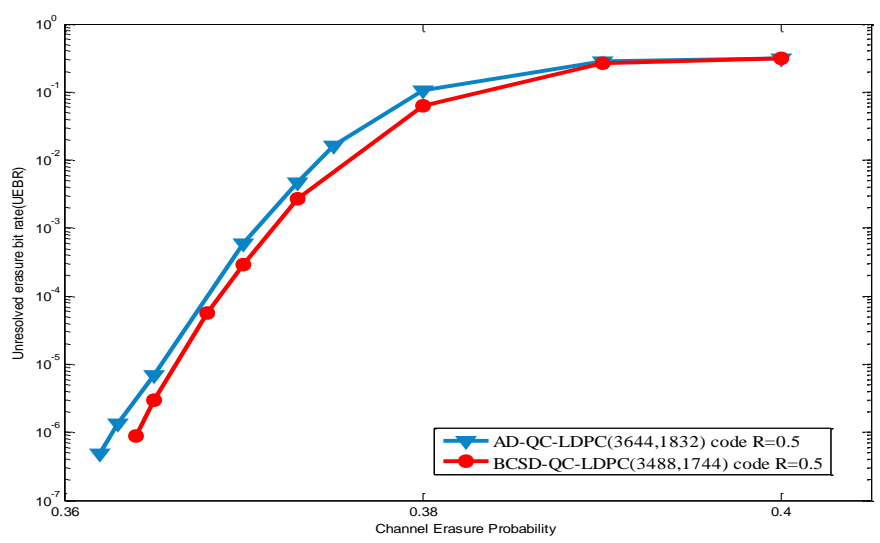

Figure 4. Performance of BCSD-QC-LDPC $(3488,1744)$ Code for BBEC, Code Rate $=0.5$ 
Then, we compare BCSD-QC-LDPC $(8802,7335)$ code with ALDPC $(8766,7305)$ code of the classical array codes from lecture [16](zero-covering-span of the $\operatorname{ALDPC}(8766,7305)$ code is less than the zero-covering-span of the BCSD-QCLDPC $(8802,7335)$ code). From Figure 5, we can see the BCSD-QC-LDPC (8802, $7335)$ code perform better than ALDPC $(8766,7305)$ code for BBEC.

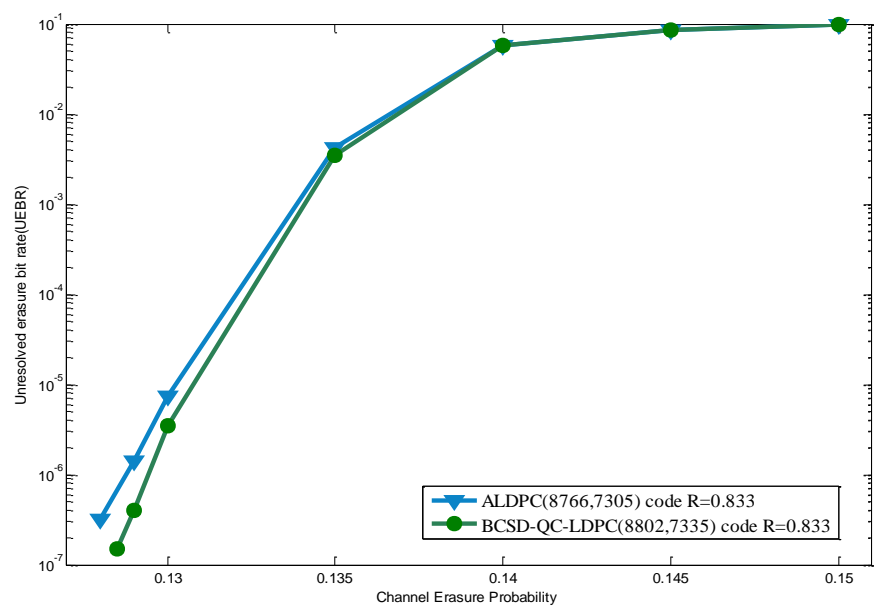

Figure 5. Performance of BCSD-QC-LDPC $(8802,7335)$ Code Compared with the ALDPC $(8766,7305)$ Code for BBEC

\section{Conclusion}

In this paper, we present a novel method based on BIBD and BCSD to construct good QC-LDPC codes for correcting erasure-bursts. We compared the BCSD with the AD about its shortest code length with same code rate. The proposed method possesses flexible code length code rate and the decoding performance of the codes are very close to Shannon limit over the BBEC. On the other hand, the zerocovering-span of the BCSD-QC-LDPC codes are bigger than the AD-QC-LDPC codes with same code rate. It means the BCSD-QC-LDPC codes have stronger ability for correcting erasure-bursts.

The simulation results show that, the performance of BCSD-QC-LDPC codes perform better than BIBD-QC-LDPC codes, BIBDdes -QC-LDPC codes, AD-QCLDPC codes and the ALDPC codes with similar code length over BBEC.

\section{Acknowledgements}

This work is supported by the National Natural Science Foundation of China (61371096, 61171158, 61275077), the Natural Science Foundation Project of CQ CSTC (cstc2013jcyjA40052, cstc2012jjA40060), the Scientific and Technological Research Program of Chongqing Municipal Education Commission (Grant No.KJ130515).

\section{Reference}

[1] L. Y. Sui, J. Y. Fu and X. H. Yang, "Forward error correction", Proceedings of the 4th International conference of Computational and Information Sciences (ICCIS),(2012) August, pp. 37-40, New York, America.

[2] R. G. Gallager, "Low-density parity-check codes", IRE Transactions on Information Theory, vol. 8, (1962), pp. 21-28.

[3] G. H. Zhang, R. Sun and X. M. Wang, "Construction of girth-eight QC-LDPC codes from greatest common divisor" , IEEE Communications Letters, vol. 17, (2013),pp. 369-372.

[4] Y. G. Wang, S. C. Draper and J. S. Yedidia, "Hierarchical and high-girth QC LDPC codes", IEEE Transactions on Information Theory, vol. 59, (2013), pp. 4553-4583. 
[5] P. Hosung, "Design of Multiple-Edge Protographs for QC LDPC Codes Avoiding Short Inevitable Cycles", IEEE Transactions on Information Theory, vol. 59, (2013), pp. 4598-4614.

[6] D. J. C. Mackay, "Good error-correcting codes based on very sparse matrices", IEEE Transactions on Information Theory, vol. 45, (1999), pp. 399-431.

[7] D. Diteter, J. B. Joseph, "Ful 1 diversity Random LDPC codes", Symposium of the 18th IEEE Communications and Vehicular Technology in the Benelux (SCVT), (2011) November, pp. 1-6, Ghent, Belgium.

[8] B. N. Vellambi and F. Fekri, "Results on the improved decoding algorithm for low-density parity-check codes over the binary erasure channel", IEEE Transactions on Information Theory, vol. 53, (2007), pp. $1510-1520$.

[9] M. G. Luby, "Practical loss-resilient codes", Proceedings of the twenty-ninth annual ACM symposium of Theory of computing, (1997), pp. 150-159, New York, America.

[10] C. Y. Di ,"Finite-length analysis of low-density parity-check codes on the binary erasure channel" , IEEE Transactions on Information Theory, vol. 48, (2002), pp.1570-1579.

[11] L. Lan, "New constructions of quasi-cyclic LDPC codes based on special classes of BIBD's for the AWGN and binary erasure channels", IEEE transactions on communications, vol. 56, (2008), pp. 39-48.

[12] D. R. Stinson, "Combinatorial designs: construction and analysis", Springer, New York, (2004).

[13] W. Ryan and S.Lin, "Channel codes: classical and modern", Cambridge University Press, Oxford (2009).

[14] J. G. Yuan, C. Y. Li and S. Huang, "A novel construction method of QC-LDPC codes for optical communications based on the BIBD and circulant decomposition", Journal of Optoelectronics •Laser, (2013), pp. 9-12.(in Chinese).

[15] L. Zhang and S. Lin, "Quasi-cyclic LDPC codes on cyclic subgroups of finite fields." IEEE Transactions on Communications, vol. 59, (2011), pp. 2330-2336.

[16] J. L. Fan. "Array codes as LDPC codes." Constrained Coding and Soft Iterative Decoding, (2001), pp. 195-203

\section{Authors}

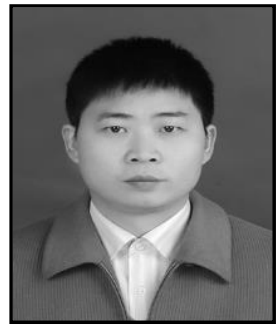

Sheng Huang, received his M.S. degree in communication and information system from Huazhong University of Science and Technology in 2003, received his Ph.D. degree in Electrical Circuit and system from Chongqing University in 2008. He is now a professor at School of Communication and Information Engineering, Chongqing University of Posts and Telecommunications. His research interests include optical communication system, channel coding and networks.

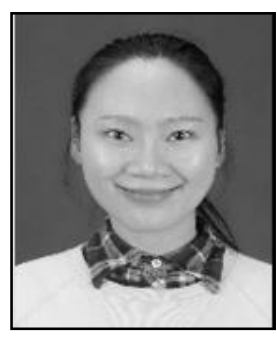

Xueting Jia, she is currently pursuing the M.S. degree with the Department of Information and Communication Engineering in Chongqing University of Posts and Telecommunications (CQUPT). Her main research interests include optical communication system, channel coding and information theory.

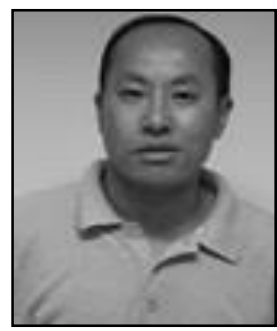

Jianguo Yuan, was born in Chongqing city, China, in 1968. He got his Ph.D. degree in Chongqing University (CU) in 2007. His doctoral paper was awarded the excellent graduation paper. He is now a professor at School of Communication and Information Engineering, Chongqing University of Posts and Telecommunications. His main research direction concerns the optical communication system, channel coding. 


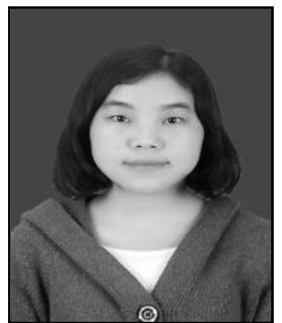

Pan Mu, she got her B.S. degree from the Chongqing University of Posts and Telecommunications (CQUPT). She is currently pursuing the M.S. degree with the Department of Information and Communication Engineering in CQUPT. Her main research interests include optical communication system, channel coding and information theory. 